Ann. Sci. forest., I976, 33 (2), 87-I07.

\title{
PRODUCTION DE LITIÈRE ET APPORT AU SOL D'ÉLÉMENTS MINÉRAUX DANS QUELQUES PEUPLEMENTS RÉSINEUX DE BRETAGNE
}

\author{
J. C. GLOAGUEN et J. TOLFIET \\ Laboratoire de Cryptogamie et d' Écologie végétale, \\ Complexe scientifique Beaulieu, \\ 35031 Rennes Cedex
}

RÉSUMÉ

La production annuelle de litière et l'apport au sol d'éléments minéraux (azote, calcium, potassium, magnésium, phosphore, manganèse, sodium, fer et cuivre) ont été déterminés dans des plantations de 8 espèces de Conifères de 20 à 33 ans : Pinus sylvestris, Pinus nigra laricio, Pinus murrayana, Picea abies, Picea sitchensis, Pseudotsuga menziesii, Tsuga heterophylla et Abies grandis, au cours de 4 années : 1971, 1972, I973 et 1974.

\section{IN'TRODUC'TION}

L'introduction des premières essences résineuses en Bretagne est déjà ancienne mais elle a pris une importance considérable depuis une vingtaine d'années. Aux Pins maritimes et aux Pins sylvestres se sont ajoutées un bon nombre d'espèces, surtout d'origine Nord-américaine, dont la croissance est particulièrement intéressante. Mais à côté de cet avantage économique indéniable, on connaît mal l'influence de la nature et de la quantité de litière qui retourne au sol sur d'une part, les caractéristiques de 1'humus et les processus de pédogenèse et, d'autre part, la nutrition et la croissance des arbres. Certains écologistes s'inquiètent, en particulier sous le climat pluvieux de Bretagne, de l'action des litières résineuses sur l'évolution des sols.

De nombreux travaux concernant la production de litière, et plus généralement le cycle des éléments minéraux dans les écosystèmes forêts, ont été réalisés depuis la fin du siècle dernier, dont des synthèses ont été données par divers auteurs : 
Lutz et Chandler (i946), Ovington (i962), Bray et Gorham (i964) et Rodin et BAzILEVICH (I967). Nous ne citerons ici, parmi les travaux les plus récents, que ceux de Carlisle, Brown et White (ig66) en Angleterre, Delecour, Weissen et Nanson (ig67), Duvigneaud et Denaeyer-De-SMet (I970) et Gossuin (I974) en Belgique, Nihigåd (I972) en Suède, Ulrich et MAYER (I973) en Allemagne, Alvera (i973) en Espagne, Cole, Gessel et Dice (I967) aux États-Unis, Militer (ig68) et Wili. (I968) en Nouvelle-Zélande, Tsu'ssumi (i97i) au Japon.

En France, nous noterons les études de GuiT'TéT (ig67) sur le Pin sylvestre, de Rapp (I967, i969) et Lossaint et Rapp (I97I) sur le Pin d'Alep et les Chênes vert et kermès, de Lanier (I g68) sur le Pin sylvestre, de Toutain et Duchaufour (I970) sur le Hêtre, de Lemés et Bichaut (I97I) sur le Hêtre seul ou en mélange avec le Chêne sessile et de Aussenac (ig69) puis Aussenac, Bonveau et Le Tacon (I972) sur le Pin sylvestre, l'Épicéa commun, le Sapin de Vancouver et un peuplement mélangé de Hêtre et de Charme.

Notre étude a pour but de comparer la production de litière et l'apport au sol d'éléments biogènes dans divers peuplements résineux. Elle a été effectuée au cours des années I97I, I972, I973 et I974, dans des reboisements situés les uns en forêt de Villecartier (Ille-et-Vilaine) où de semblables travaux ont été réalisés sur la Hêtraie (GLOAGUEN et ToufFers, 1974), les autres à quelques kilomètres en bordure Sud-Ouest de la forêt au lieu-dit le Bois-Robert, commune de Bazougesla-Pérouse (Ille-et-Vilaine).

\section{LES STATIONS ÉTUDIÉES}

Les caractéristiques générales de la région ont déjà été présentées à plusieurs

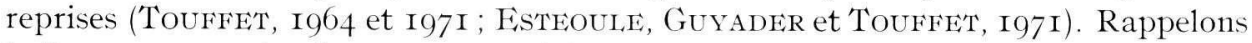
brièvement que le climat est tempéré océanique de type breton à hiver doux (un à trois mois seulement de température inférieure à $5^{\circ} \mathrm{C}$ ) et à été frais (moyennes thermiques mensuelles des mois chauds comprises entre $\mathrm{I} 5^{\circ}$ et $\left.\mathrm{I} 9^{\circ} \mathrm{C}\right)$. On y dénombre, d'après les données I95I-I970 de la station météorologique d'Antrain (Ille-et-Vilaine) située à $4 \mathrm{~km}$ de la forêt, I 40 jours de pluie par an pour une hauteur annuelle des précipitations de $896 \mathrm{~mm}$ régulièrement réparties tout au long de l'année.

La durée moyenne de l'insolation est d'après LARIVIÈre et Verdou (ig69) de I 900 heures par an, ce qui correspond à un nombre d'heures d'ensoleillement moyen pour le Nord de la Bretagne.

Le substrat géologique est le massif granitique d'âge cadomien dit de Bonnemain (d'après la carte géologique de la France au I/80 $000^{\circ}$, feuille d'Avranches), généralement altéré en surface en un sable jaunâtre.

Les sols qui en résultent sont légèrement différents suivant les stations. Ce sont des sols bruns acides à humus acide de type moder, à texture limoneuse ou limono-sableuse. Au Bois-Robert ils présentent un horizon supérieur homogène de 25 à $30 \mathrm{~cm}$, attestant d'un terrain anciennement cultivé, qui repose sur un horizon $\mathrm{B}$ de 30 à $50 \mathrm{~cm}$, légèrement hydromorphe dans la zone reboisée en Picea sitchensis.

A Villecartier, 1'hydromorphie est encore plus nette et nous avons des horizons 
à pseudogley bien marqués dans les peuplements de Picea abies et de Tsuga heterophylla.

Huit espèces de Conifères font l'objet de cette étude ; six d'entre elles sont implantées au Bois-Robert, il s'agit de Pinus sylvestris, P. nigra laricio, P. murravana, Picea sitchensis, Abies grandis et Pseudotsuga menziesii (deux parcelles ont été suivies pour cette dernière espèce), les deux autres (Picea abies et Tsuga heterophylla) en bordure de la forêt de Villecartier.

Quelques caractéristiques des peuplements étudiés sont réunies dans le tableau I.

TABLEAU I

Caractéristiques des neuf peuplemenis étudiés

Characteristics of the nine stands studied

\begin{tabular}{|c|c|c|c|c|c|c|c|}
\hline Fispèces & 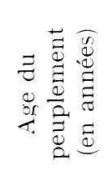 & 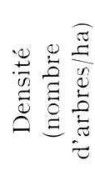 & 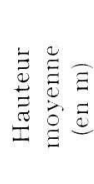 & 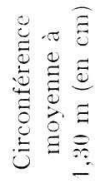 & 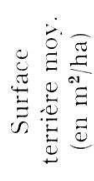 & 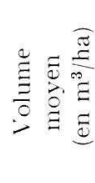 & 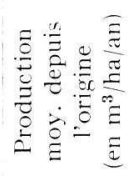 \\
\hline Pinus sylvestris & 20 & 2190 & 13,0 & 40,1 & $28,1)$ & 152,6 & 7,6 \\
\hline Pinuss nigra laricio & 20 & 1850 & $1: 2,5$ & 40,0 & 23,6 & $12: 3,3$ & 6,2 \\
\hline Pinus murrayana & 20 & 1750 & 13,0 & 54,6 & 41,5 & $2 \cdot 24,1$ & 11,3 \\
\hline Picea sitchensis & $\because 0$ & -258 & $1 \because, 5$ & ix, & 17,7 & $2 \div 9,7$ & $1: 2,5$ \\
\hline Picea abies & 33 & $1: 281$ & 20,0 & 59,1 & 35,6 & $-298,1$ & 9,0 \\
\hline T:uga heterophylla & 24 & 1830 & 15,0 & 57,0 & 47,3 & 297,3 & $12,{ }^{\prime}$ \\
\hline Pseudotsuga menziesii I & 20 & $\because 220$ & 12,5 & 38,5 & 26,2 & 137,1 & 6,9 \\
\hline Pseudotsuga menziesii $\mathrm{II}$ & 20 & 2530 & 13,5 & 12,3 & 36,0 & 203,7 & $10, \geq 2$ \\
\hline Ahies grandis & 20 & 2.290 & 13,5 & 47,9 & 41,8 & 236,4 & 11,8 \\
\hline
\end{tabular}

\section{LA PRODUCTION ANNUELIE DE LITIÈRE}

La litière est recueillie dans I 2 cagettes de $54 \times 32,5 \mathrm{~cm}$ disposées au hasard à la surface du sol de chaque peuplement et ramassée tous les trois mois : fin décembre, fin mars, fin juin et fin septembre, les récoltes trimestrielles correspondent ainsi pratiquement à la chute de litière pendant les quatre saisons.

Le matériel, trié, est mis à $1^{\prime}$ étuve à $65^{\circ} \mathrm{C}$ puis pesé $\left.{ }^{1}\right)$.

(1) Toutes les valeurs indiquées sont mesurées ou calculées après séchage a $65^{\circ} \mathrm{C}$. A cette tempé rature, l'humidité résiduelle par rapport au poids sec à $105^{\circ} \mathrm{C}$ est comprise entre 2,5 et 3 p. 100. 


\section{TABLEAU 2}

Production annuelle de litière dans les neuf peuplements étudiés (en $\mathrm{g} / \mathrm{m}^{2}$ ) Yearly litter production in the nine stands studied $\left(\mathrm{g} / \mathrm{m}^{2}\right)$

\begin{tabular}{|c|c|c|c|c|c|c|}
\hline Espèces & Années & Aiguilles & Brindilles & Cònes & Divers & Total \\
\hline \multirow{5}{*}{ Pinus sylvestris } & 1971 & 672,3 & 23,9 & 11, t $^{\prime}$ & 25,3 & $73:, 9$ \\
\hline & 1972 & 676,1 & 59,6 & $1: 2,9$ & $\uparrow 3,9$ & 792,5 \\
\hline & 1973 & 557,1 & 58,5 & $16,,^{\prime}$ & $6.5,1$ & 697,1 \\
\hline & 1974 & 736,2 & 215,6 & 29,0 & 911,9 & 1071,7 \\
\hline & Moyenne & 660,4 & 89,4 & $1 i, t$ & 56,3 & 823,5 \\
\hline \multirow{5}{*}{ Pinus nigra laricio } & 1971 & 497,5 & 30,8 & - & $s,+j$ & $536 ;, 9$ \\
\hline & 1972 & 555,0 & 18,5 & $6, \underline{-2}$ & 27,0 & 606,7 \\
\hline & 1973 & 425,7 & 8,4 & 2,6 & 10,11 & 176,7 \\
\hline & 1974 & 722,2 & 5,9 & 16,9 & 47,2 & $79 \cdot 2,2$ \\
\hline & Moyenne & 550,1 & 15,9 & 6,4 & $30, i$ & 1003,1 \\
\hline \multirow{5}{*}{ Pinus murrayana } & 1971 & 561,9 & 17,3 & $: 33,7$ & 36,0 & $66^{\prime}+8,9$ \\
\hline & 1972 & 718,4 & 52,7 & 16,1 & $5 \times, \overline{7}$ & 875,9 \\
\hline & 1973 & 519,2 & î, & $9=2,8$ & $76, \overline{7}$ & $7 \geq 9,1$ \\
\hline & 1974 & 801,3 & $10 /, 8$ & $166^{\prime}, 7$ & {$[115,5$} & $1176, .3$ \\
\hline & Moyenne & $6 ; 50,2$ & 53,8 & 84,3 & 69,2 & $85 i, 5$ \\
\hline \multirow{5}{*}{ Picea sitchensis } & 1971 & 583,0 & 1,1 & - & 7,6 & 591,7 \\
\hline & $197-2$ & 916,9 & 7,1 & - & 17,8 & $9^{\prime}+2,1$ \\
\hline & 1973 & 483,7 & 6,1 & - & 10,5 & $500(, 3$ \\
\hline & 1974 & 748,3 & 22,3 & 2,11 & $1 \times, 9$ & 791,5 \\
\hline & Moyenne & 683,0 & 9,2 & 0,5 & 13,2 & $\therefore 06, t$ \\
\hline \multirow{5}{*}{ Picea abies } & 1971 & 603,0 & 22,0 & - & $1:, 5$ & $637 \overline{5} 5$ \\
\hline & 1972 & 1315,9 & 49,4 & 一 & $\because 4, j$ & 1389,8 \\
\hline & 1973 & 251,3 & 18,0 & - & 26,1 & 295,7 \\
\hline & 197' & 538,3 & 59,2 & - & $27,1)$ & $6 i 2^{\prime}, 5$ \\
\hline & Moyenne & $6 z i, 1$ & $3 \pi, 2$ & - & $2: 2,6$ & 736,9 \\
\hline \multirow{5}{*}{ Tsuga heteroplivlla } & 1971 & 309,8 & 3,1 & - & $\ddot{2}, x$ & 315,7 \\
\hline & $197: 2$ & $2: 28,7$ & 3,0 & - & $7, \underline{2}$ & 238,9 \\
\hline & 1973 & 285,3 & 4,6 & 0,3 & 3,9 & $299_{4}^{\prime}, 1$ \\
\hline & 1974 & 291,9 & 15,6 & & 8,$1 ;$ & 316,1 \\
\hline & Ioyenne & 278,9 & 6,6 & 0,1 & 5,6 & 291,2 \\
\hline \multirow{4}{*}{ Pseudotsuga mensiesii I } & 1971 & 319,2 & -5 & + & $\therefore-$ & , \\
\hline & 1972 & 312,5 & 2,1 & - & 11,2 & $\begin{array}{l}328,4 \\
3 \div 7,8\end{array}$ \\
\hline & 1973 & $-275,8$ & 10,2 & - & 13,15 & 299,6 \\
\hline & Moyenne & 202,5 & 13,3 & & 9,5 & 325,3 \\
\hline \multirow{3}{*}{ Pseudotsuga menziesii II } & 1973 & 361,9 & 9,5 & - & 7,6 & $3 ; 9,0$ \\
\hline & 1974 & $4-99,3$ & 22,4 & - & $10, x$ & $16 \geq 2,5$ \\
\hline & Moyenne & 395,6 & 16,0 & - & 9,2 & 420,8 \\
\hline Abies grandis & 1974 & 532,7 & 3,1 & - & 17.9 & 553,7 \\
\hline
\end{tabular}




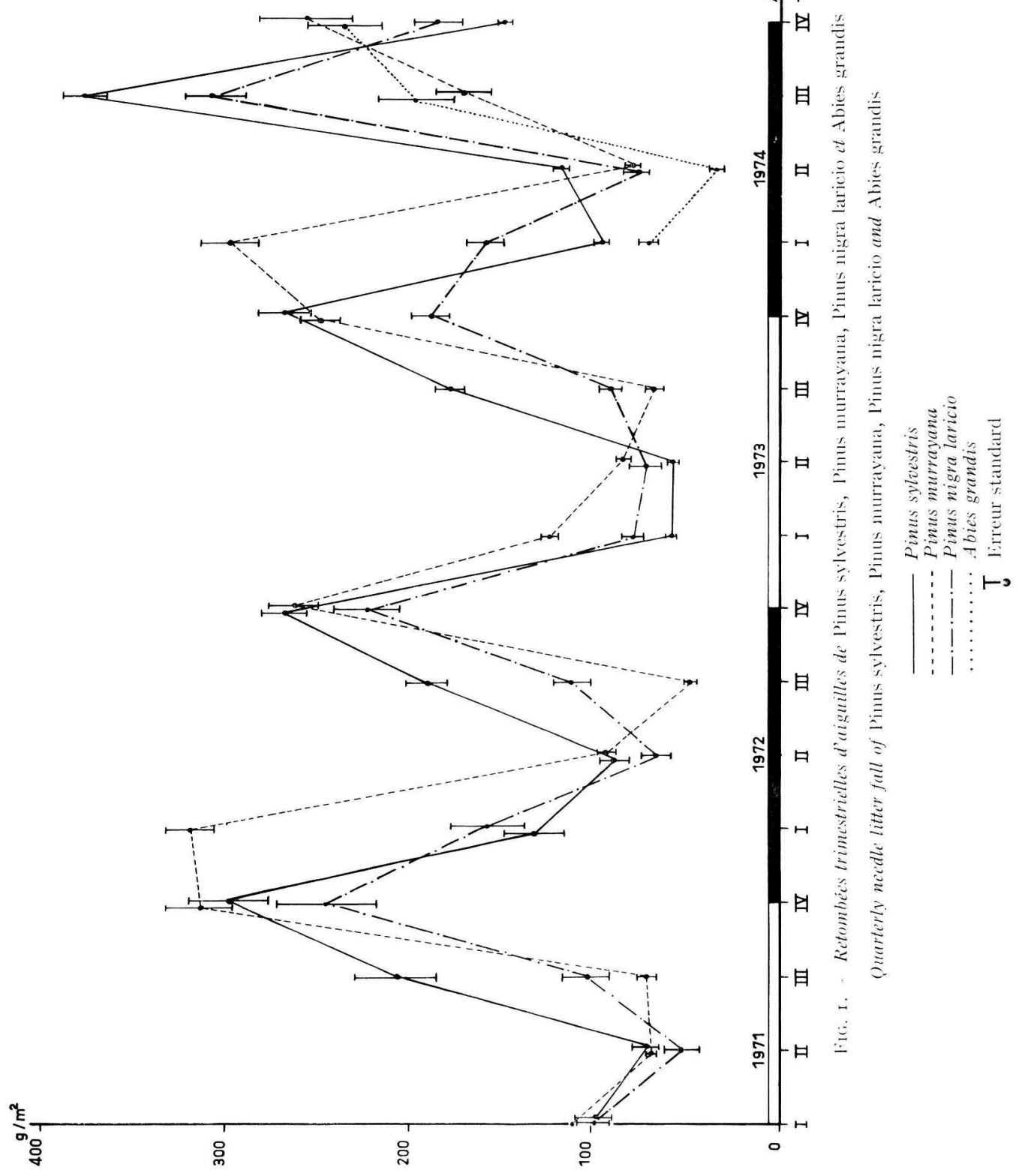




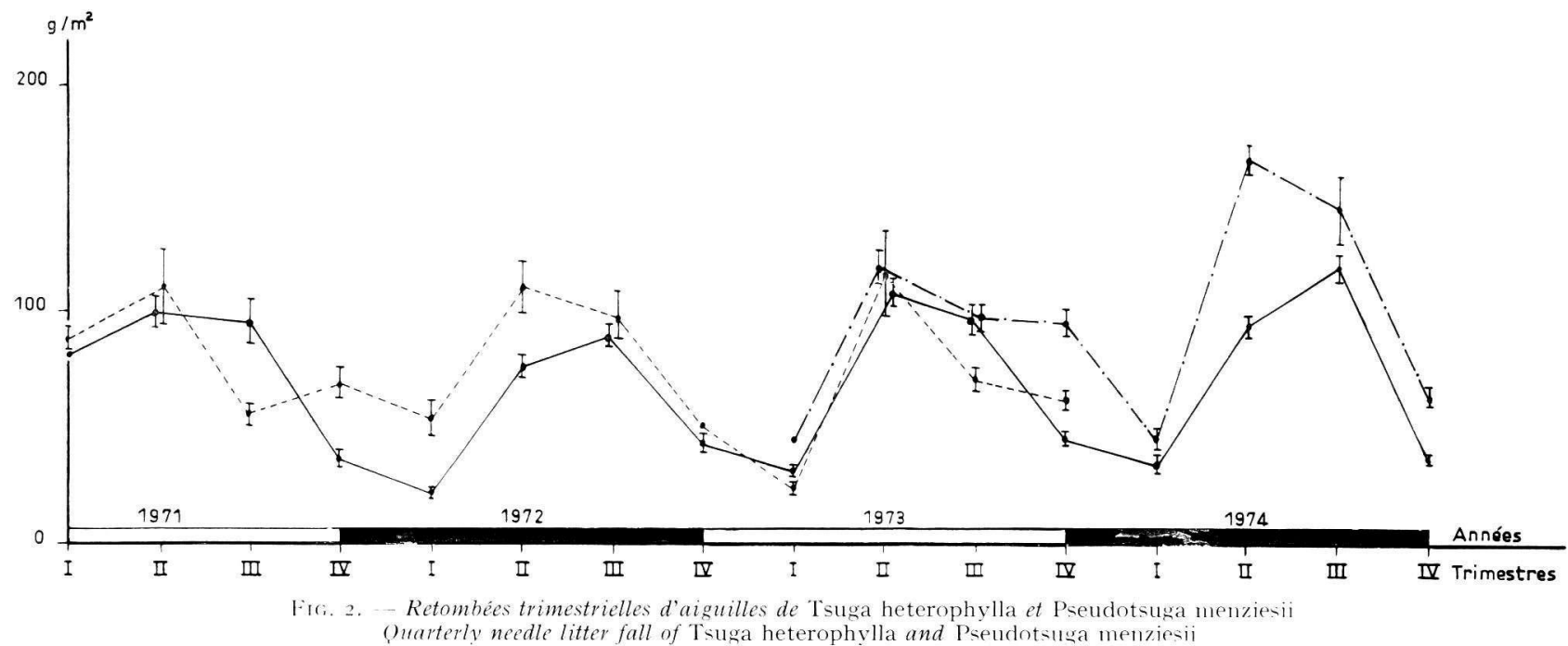
Ouarterly needle litter fall of Tsuga heterophylla and Pseudot:uga menziesii

$$
\begin{aligned}
& \text { _..... Psuga heterophylla } \\
& \text { _._-._- Pseudotsuga menziesil II } \\
& \text { T. Erreur standard }
\end{aligned}
$$


Les résultats obtenus sont regroupés dans le tableau 2. Nous y avons noté les poids des aiguilles, des brindilles, des cônes et des divers (débris d'aiguilles, morceaux d'écorce et parfois écailles de bourgeons et chatons).

Les figures I, 2 et 3 représentent les chutes trimestrielles d'aiguilles au cours des trois années d'étude.

Parfois une ou plusieurs cagettes ont été déplacées, renversées ou volées; dans: certains cas, c'est même l'ensemble de la série qui a été emporté (la valeur manquante est alors calculée par la formule de YATEs (in SNEDECOR et Cochran, I97I) et représentée par un simple point dans les figures I, 2 et 3 , sans indication de l'erreur standard).

Fin moyenne, chez toutes les espèces l'erreur standard est inférieure à Io p. Ioo. le nombre de 12 cagettes peut ainsi être considéré comme raisonnable. Néanmoins elle est nettement supérieure à celle de la Hêtraie où elle était dans la plupart des cas comprise entre 2 et $4 \mathrm{p}$. Ioo en forêt de Villecartier pour un même nombre de cagettes (Gloaguen et Tourfert, I974).

Aussenac (rg69) constatait la même différence entre les peuplements feuillus et résineux de l'Esst de la France.

Parfois l'erreur standard est cependant plus élevée (elle atteint même une foi20 p. IOO), il s'agit assez souvent de périodes où la chute de litière est faible, mai il est impossible de généraliser.

La précision des moyennes pour la litière totale est sensiblement la même que pour les aiguilles, mais par contre, pour les brindilles et les cônes dont la chute est beaucoup plus irrégulière, l'erreur standard est beaucoup plus élevée : les valeurs: indiquées ne représentent donc que des ordres de grandeur.

Chez toutes les espèces, les valeurs obtenues pour la chute annuelle de litière (tab1. 2) sont relativement élevées si nous les comparons aux données citées par Bornebusch (I937), Mork (I942), Tarrant, Isaac et Chandler (I951), OWen (I954), Viro (i955), Bonnevie-Svendsen et Gjems (i957), Ovington (i959), Heilman et Gessel (i963), Bray et Gorham (ig64), Rodin et Bazilevich (ig67), AusseNAC (I969), NiHLGår) (I972) et Gossuin (I974), d'autant plus qu'elles sont très légèrement sous-estimées, la décomposition des aiguilles étant déjà amorcée lors de leur récolte. Ces fortes valeurs peuvent s'expliquer par la densité des plantations qui n'ont jamais été exploitées et qui sont installées sur des sols relativement riches. Rodin et BAzILEvich ( 1967 ) notent que la production annuelle de litière d'aiguilles des Épicéas est supérieure à celle des autres Conifères ; cela se vérifie pour les espèces étudiées que nous pouvons classer dans l'ordre suivant d'après les moyennes. obtenues :

Picea sitchensis $>$ Picea abies $>$ Pinus sylvestris $>$
Pinus murrayana $>$ Pinus nigra laricio $>$ Abies grandis
Pseudotsuga menziesii $>$ Tsuga heterophylla

La différence entre les 2 peuplements de Pseudotsuga menziesii semble être due aux origines différentes des graines.

Pour la production annuelle de litière totale l'ordre des espèces les plus productives est modifié notamment par les brindilles et les divers mais aussi par les cônes, les Pins étant plus ou moins fertiles alors que les autres résineux ne le sont pratiquement pas ; le classement devient le suivant : 


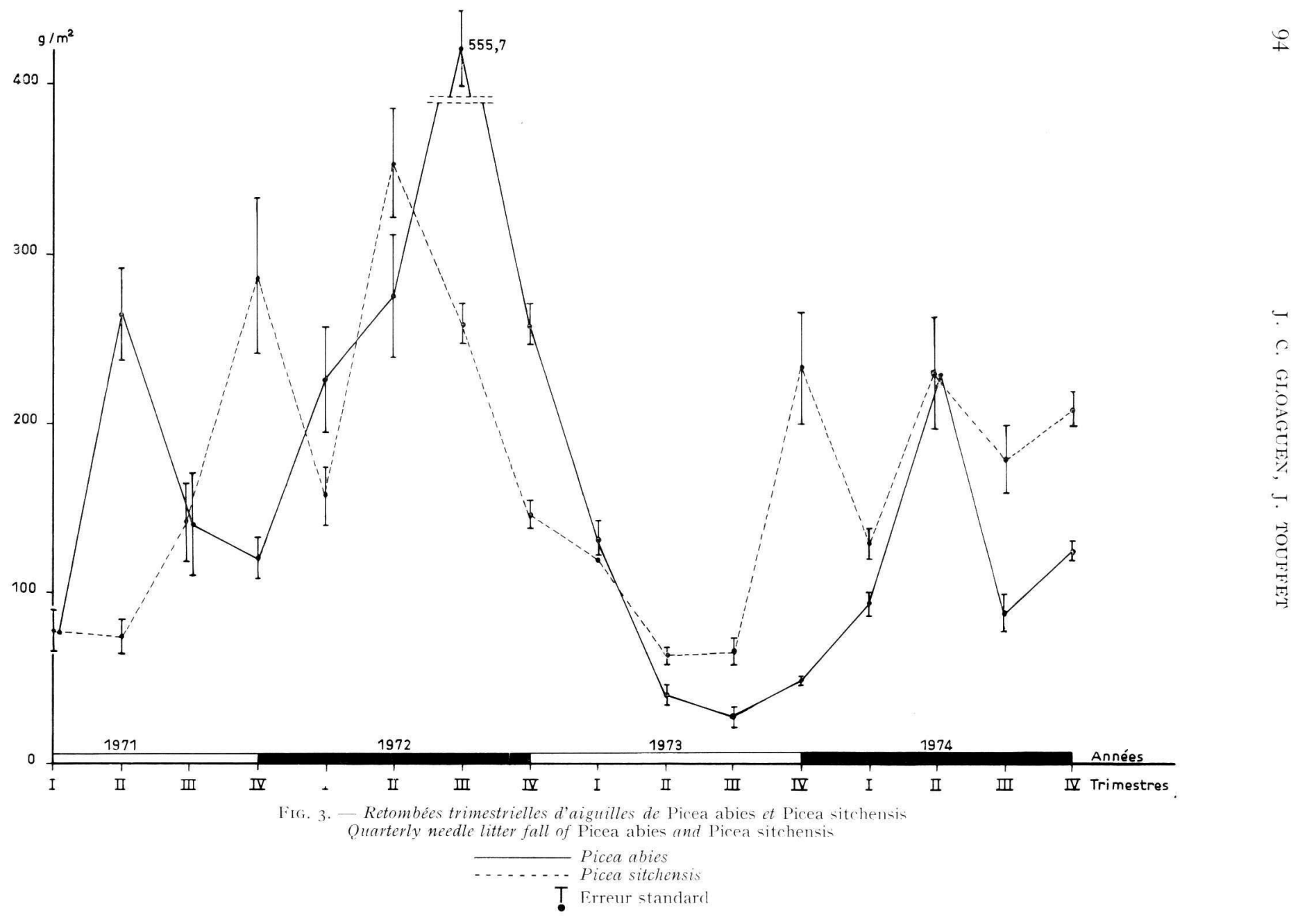


Pinus murrayana > Pinus sylvestris $>$ Picea abies $>$

Picea sitchensis > Pinus nigra laricio > Abies grandis > Pseudotsuga menziesii $>$ Tsuga heterophylla.

Les variations de production de litière d'aiguilles d'une année à l'autre sont très inégales selon les espèces (fig. 4) : elles sont faibles pour les espèces les moins productives, fortes pour les plus productives et notamment pour Picea abies. Les différences annuelles pourraient dépendre des précipitations : RODIN et BAzILEVICH (I967) remarquent que la production de litière d'aiguilles est plus faible les années sèches que les années humides; cela est vrai pour la plupart des espèces si nous comparons I97I, I973 et I974 (fig. 4), mais I972 vient contredire cette assertion.

L'observation des figures $I, 2$ et 3 représentant les chutes trimestrielles d'aiguilles nous montre que les diverses espèces étudiées ne se comportent pas de la même manière.

Il est possible d'en distinguer 3 groupes :

I. Pinus sylvestris, Pinus nigra laricio, Pinus murrayana et Abies grandis (fig. I), dont la chute d'aiguilles est maximale en automne (en moyenne sur 4 ans).

Pour Pinus murrayana, l'abondance des chutes d'aiguilles en fonction des saisons obéit à l'ordre suivant :

$$
\text { Automne }>\text { Hiver }>\text { Été }>\text { Printemps. }
$$

Pour les 3 autres espèces, l’ordre est légèrement différent :

$$
\text { Automne }>\text { Été }>\text { Hiver }>\text { Printemps. }
$$

2. Tsuga heterophylla et Pseudotsuga menziesii (fig. 2), dont la chute est maximale au printemps ou en été.

Ainsi, chez Tsuga, en moyenne, l'ordre est le suivant:

$$
\text { Été }>\text { Printemps }>\text { Automne }>\text { Hiver }
$$

et pour Pseudotsuga :

$$
\text { Printemps }>\text { Été }>\text { Automne }>\text { Hiver }
$$

3. Picea abies et Picea sitchensis (fig. 3), qui ont une chute d'aiguilles très irrégulière.

Cette opposition entre espèces dont la chute d'aiguilles est soit saisonnière soit irrégulière a été observée par de nombreux auteurs, mais les résultats obtenus sont parfois contradictoires (voir notamment BRAY et GoRHAM, I964).

Ainsi pour Picea abies, Chandler (I944) note une chute d'aiguilles plus faible au printemps et en été au N.-E. des États-Unis, et pour NiHLGÅRD (I972), en Suède, la production de litière est également plus faible en été ; par contre LiNDBERG et Norming (in Bray et Gorham, I964), en Suède aussi, constatent un plus fort taux de chute fin printemps-début été, tout comme BorNeBusch (I937) au Danemark, pour qui la plus grande chute naturelle a lieu dans la période de mi-mars à fin août. Aussenac (I969) et Gossuin (1974) observent un maximum en automne avec un maximum secondaire au printemps mais le premier auteur souligne, comme 


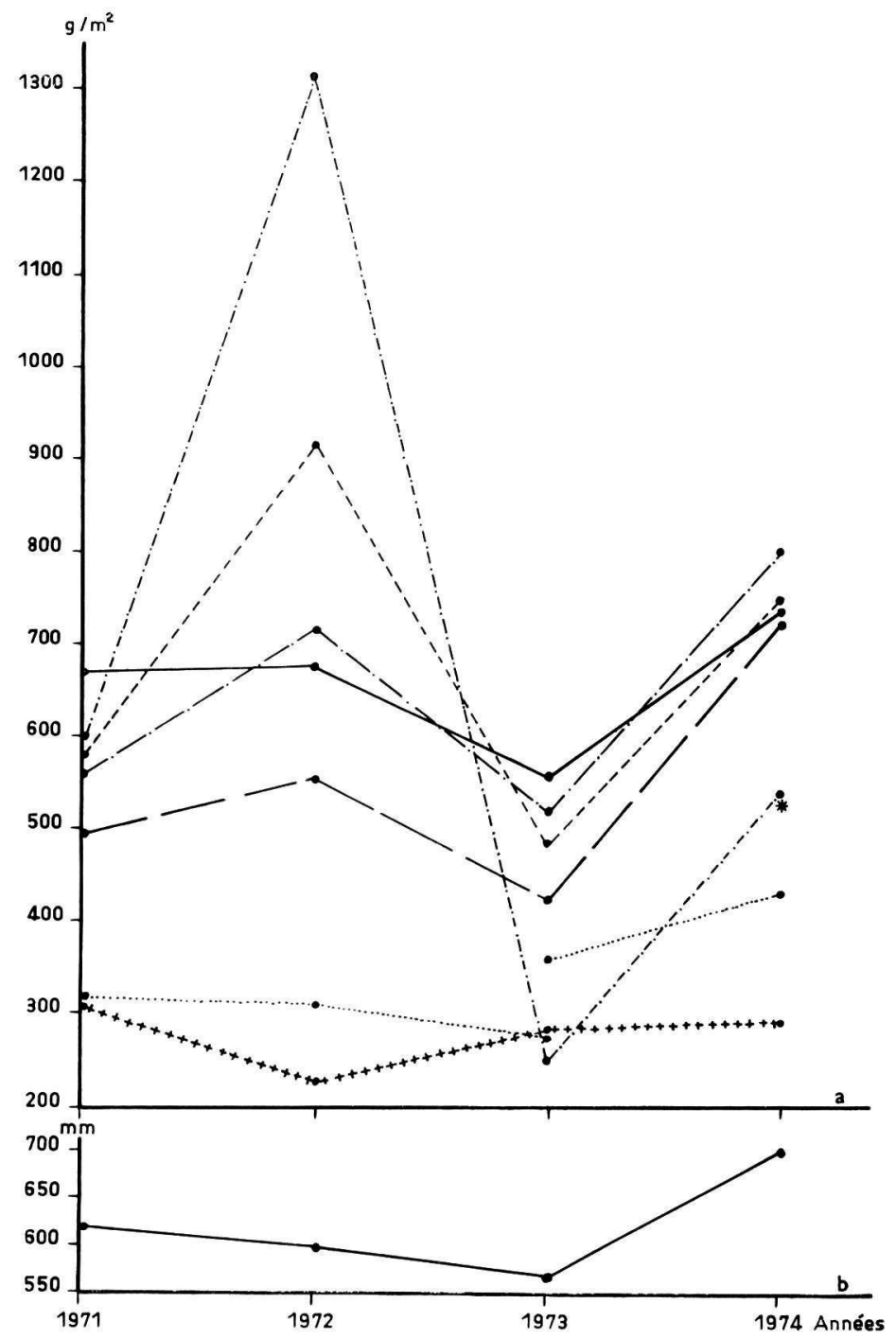

İIG, 4. - Retombées annuelles d'aiguilles (a)

ct pluviosité annuelle à Rennes-Saint-Jacques (b) au cours des quatre années d'étude

Searly needle litter fall (a) and yearly rainfall in Rennes-Saint-Jacques (b) during the four-year study

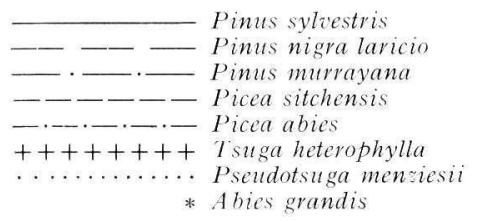


BORNEBUSCH (I937), qu'une sécheresse estivale peut provoquer une chute importante d'aiguilles. Nous constatons le même phénomène en I972, dont le printemps et l'été furent les plus secs des 4 années d'étude : la production en été I972 fut exceptionnelle, or on sait que la sécheresse favorise 1'abscission (in ADDIcotr et LYON, I973).

Comme OWEN (1954) au Pays de Galles, notons pour Picea sitchensis une légère tendance vers 2 maximums : l'un au printemps, l'autre en automne, ce dernier nous paraissant plus net.

Enfin Viro (I955) et Alvera (I973) pour Pinus syliestris et Ausserac (I969) pour Pimus sylvestris et Abies grandis, signalent une retombée maximale à la fin de l'été et en automne; nous arrivons à la même conclusion chez ces 2 espèces où l'automne est la saison de plus forte chute, suivie de l'été.

\section{LA RESTITUTION D'ÉLÉMENTS MINÉRAUX AU SOL, PAR LES LITIÈRES}

\section{I. - Méthodes d'analyse}

Les échantillons à analyser (aiguilles, brindilles, cônes) sont broyés au Dangoumeau après passage à l'étuve à $65^{\circ} \mathrm{C}$.

Les poudres sont traitées par minéralisation nitroperchlorique.

Les dosages d'éléments (sauf pour l'azote) se font sur les solutions ainsi obtenues:

- le potassium et le sodium au photomètre à flamme ;

- le calcium, le magnésium, le fer, le manganèse et le cuivre au spectrophotomètre d'absorption atomique ;

- le phosphore au photocolorimètre, en présence de molybdate d'ammonium (réduction à l'acide ascorbique: DUVAL, Ig62).

La teneur en azote est déterminée après minéralisation au micro-kjeldahl par la technique de Parnas et Wagner.

\section{II. - Composition minérale}

Les aiguilles recueillies chaque trimestre au cours des années I97I, I972, I973, 1974, ont été analysées. Les moyennes trimestrielles pour les 4 années sont reportées dans le tableau 3 .

D’une façon générale, il apparaît que les teneurs en éléments biogènes décroissent dans 1'ordre suivant :

$$
\mathrm{N}>\mathrm{Ca}>\mathrm{K}>\mathrm{Mg}>\mathrm{P}>\mathrm{Mn}>\mathrm{Na}>\mathrm{Fe}>\mathrm{Cu} .
$$

Les valeurs obtenues s'intègrent dans les données, d'ailleurs très variables, citées par divers auteurs comme OWEN (I954), VIRO (I955), BONNEVIE-SVENDSEN et Gjems (I957), Rodin et Bazilevich (I967), Aussenac, Bonneau et Le Tacon (1972), avec souvent des valeurs plus faibles pour le calcium, conséquence de la nature granitique du substrat. 
TABLE,AU 3

Teneurs (en p. Ioo du poids sec) en éléments minéraux des aiguilles de la litière Movenne trimestrielle des années 1971, 1972, 1973 et 1974

Contents (in p. Ioo of dry weight) of mineral elements in the needle litter fall Quarterly average of $1971,1972,1973$ and $19 \% 4$

\begin{tabular}{|c|c|c|c|c|c|c|c|c|c|c|}
\hline Escence & Trinn. & $\lambda$ & $\mathrm{Ca}$ & K & $M g$ & $\mathrm{I}^{\prime}$ & Mn & $\mathrm{Na}$ & $\mathrm{Fe}$ & $\mathrm{Cu}$ \\
\hline \multicolumn{11}{|l|}{-} \\
\hline \multirow{4}{*}{ Pinus syloestris } & $1 \mathrm{er}$ & 1,26 & 0,51 & $0, \underline{2}$ & $0,08: 3$ & $0,0.86$ & 0,0128 & 0,037 & $0,02-25$ & 0,00007 \\
\hline & $\underline{2}$ & 1,41 & 0,45 & 0,28 & (0,08:3 & 0,106 & $0,0 \geq 6$ & 0,018 & 0,030 & 0,0010 \\
\hline & $3^{\mathrm{e}}$ & 1,10 & 0,48 & 0,27 & 0,096 & $0,0) 9_{1}^{\prime}$ & $0,0: 3:$ & $0,0.25$ & 0,030 & 0,0009 \\
\hline & ie & 0,80 & 0,51 & $0,2,2$ & 0,075 & 0,0669 & $0,0 \div 1$ & $0,0 \geq 1$ & $0,02^{\prime} 1$ & 0,0010 \\
\hline \multirow{4}{*}{ Pinus nigra laricio } & 1er & $1), 81$ & 0,任 & 0,11 & 0,116 & 0,065 & 0,014 & $0,0 \geq 2$ & $0,0.27$ & 0,0010 \\
\hline & $2 e$ & 0,85 & 0,38 & 0,20 & 0,086 & 0,0170 & $0,01 \frac{1}{t}$ & $0,02: 3$ & $0,0.3^{\prime}$ & $0,001:$ \\
\hline & $3^{\mathrm{e}}$ & 11,80 & 0,37 & 0,23 & 0,112 & $0,06: 2$ & 0,015 & $0,0 \geq 6$ & $0,0 \div 21$ & 0,0009 \\
\hline & $t_{t} \mathrm{e}$ & 19,61 & 0,11 & 0,17 & 0,087 & 0,049 & 0,016 & 0,027 & 0,019 & 0,0010 \\
\hline \multirow{4}{*}{ Pinus murrayana } & $1 e r$ & 0,77 & 0,40 & $0,2,2 \cdot$ & 0,076 & 0,0664 & 0,0131 & 0,032 & $0,0.27$ & 0,0008 \\
\hline & $2 \mathrm{e}$ & (1),9' & 0,36 & 0,31 & 0,078 & 0,076 & $0,0) 25$ & 0,020 & 0,038 & 0,0012 \\
\hline & $3^{\mathrm{e}}$ & 0,86 & 0,29 & 0,29 & 0,080 & 0,078 & 0,051 & $0,0: 4$ & 0,027 & 0,0010 \\
\hline & te & 0,60 & 0,34 & $0,2,2$ & $0,068 x$ & 0,051 & 0,040 & 0,027 & 0,020 & 0,0008 \\
\hline \multirow{4}{*}{ Picea abies } & $1 \mathrm{er}$ & 0,96 & 0,35 & 0,23 & $0,18: 3$ & 0,077 & 0,013 & $0,0^{\prime} t^{\prime}{ }_{t}$ & $0,0.25$ & 0,0010 \\
\hline & 20 & 0,95 & 0,33 & $0,2,2$ & 0,135 & 0,096 & 0,016 & 0,030 & $0,0.27$ & 0,00009 \\
\hline & $3 e$ & 1,08 & 0,30 & 0,16 & $0,16: 3$ & 0,071 & 0,013 & 0,025 & 0,016 & $0,001 \pm$ \\
\hline & ite & $0,8 \geq$ & $0,3: 3$ & 0,17 & $11,1: 37$ & 0,087 & 0,017 & 0,032 & 0,025 & 0,0012 \\
\hline \multirow{4}{*}{ Picea sitchensis } & ler & 1,10 & 0,51 & 0,26 & 0,095 & 0,093 & 0,120 & 0,035 & $0,0: 32$ & 0,0009 \\
\hline & 2 & $1), 97$ & 0,61 & 0,29 & $(1,10)_{1}$ & 0,080 & 0,117 & 0,023 & $0,0.37$ & 0,0010 \\
\hline & $3^{\mathrm{e}}$ & 1,06 & 0,65 & 0,25 & 0,116 & 0,080 & 0,110 & 0,022 & $0,0.3^{\prime}$ & 0,0010 \\
\hline & ie & 0,94 & 0,52 & 0,11 & 0,111 & 0,100 & 0,104 & 0,035 & $0,0.25$ & 0,0010 \\
\hline \multirow{4}{*}{$\begin{array}{c}\text { Pseudotsuga } \\
\text { menziesii }\end{array}$} & 1er. & 1,82 & 0,58 & 0,29 & 0,143 & 0,128 & 0,128 & 0,055 & 0,042 & $0,001: 3$ \\
\hline & $\underline{x}$ & 1,62 & 0,50 & $0, \underline{y}^{\prime}$ & 0,118 & 0,116 & 0,127 & 0,032 & 0,06 ' & 0,0011 \\
\hline & $3 \mathrm{e}$ & 1,69 & 0,56 & $0, \underline{y}^{\prime}+$ & $0,13 x$ & 0,125 & 0,107 & 0,033 & $0,0.53$ & 0,0012 \\
\hline & ;e & 1,58 & 0,51 & 0,17 & 0,110 & 0,131 & 0,0194 & 0,036 & 0,043 & $0,001=$ \\
\hline \multirow{4}{*}{$\begin{array}{c}\text { Tsuga } \\
\text { heterophylla }\end{array}$} & $1 \mathrm{er}$ & 1,29 & $0, x^{\prime} 0$ & $0,4: 3$ & 0,120 & 0,096 & 0,024 & 0,077 & 0,058 & 0,0010 \\
\hline & $2 e$ & 0,82 & $0,+0$ & ו'וב, & 0,127 & 0,076 & 0,011 & 0,092 & 0,063 & 0,0010 \\
\hline & $3^{\circ}$ & 1,11 & 0,40 & 0,19 & $0,1 ' 7$ & 0,079 & 0,011 & 0,052 & 0,060 & 0,0010 \\
\hline & $4^{e}$ & 1,19 & $0,3: 3$ & 0,19 & 0,0993 & 0,114 & 0,010 & $0,0 \frac{1}{4} 0$ & 0,061 & 0,0011 \\
\hline \multirow{4}{*}{$\begin{array}{c}\text { Abies grandis } \\
\text { (197' miquement) }\end{array}$} & $1 \mathrm{er}$ & 1,66 & 0,60 & $0,2,2$ & 0,181 & 0,076 & 0,148 & $0,0 \geq 6$ & 0,040 & 0,00007 \\
\hline & $\Rightarrow e$ & 1,11 & 0,47 & 0,37 & $(1,150$ & 0,098 & $0,1,9$ & 0,025 & 0,031 & 0,0008 \\
\hline & $3^{\circ}$ & 1,39 & 0,80 & 11,26 & (1), 180 & 0,079 & 0,171 & 0,036 & 0,028 & 0,0009 \\
\hline & $4^{e}$ & 1,51 & 0,46 & 0,2 ' & (1,1:2 & 0,130 & 0,179 & 0,048 & 0,036 & 0,0008 \\
\hline
\end{tabular}

En ce qui concerne le bois et les cônes lorsqu'ils existent, seules les valeurs moyennes sont reportées dans le tableau 4. Remarquons, comme Viro (I955), la faible teneur des cônes en éléments, notamment en calcium.

a) Comparaison des diverses essences.

I1 ressort du tableau 3 que les aiguilles de Pseudotsuga menziesii ou de Abies grandis sont en moyenne les plus riches en azote, calcium, potassium, magnésium, 
phosphore; elles se différencient surtout des autres aiguilles par leurs teneurs en azote de l'ordre de I,5 p. Ioo alors qu'elles sont d'environ I p. Ioo pour les autres essences. Par contre Pinus nigra laricio et Pinus murrayana sont les moins bien pourvus en ces éléments minéraux et particulièrement en azote.

\section{TABLEAU 4}

Teneurs moyennes (en p. Ioo du poids sec) en éléments minéraux des brindilles et des cônes de la litière

Average contents (in p. Ioo of dry weight) of mineral elements in twig and cone litter fall

\begin{tabular}{|c|c|c|c|c|c|c|c|c|c|}
\hline & $\mathrm{N}$ & $\mathrm{Ca}$ & $\mathrm{K}$ & $\mathrm{Mg}$ & $\mathrm{P}$ & Mn & $\mathrm{Na}$ & $\mathrm{Fe}$ & $\mathrm{Cu}$ \\
\hline \multicolumn{10}{|l|}{ Brindilles } \\
\hline Pinus sylvestris . . . . . . & 0,61 & 0,2 't & $0,3 \div$ & 0,11 & $0,0)_{t}^{\prime}$ & 0,0055 & 0,01 ; & $0,0 \geq 2^{\prime}+$ & 0,0006 \\
\hline Pinus nigra laricio . . . . . . . & 0,80 & 0,30 & 0,13 & 0,08 & 0,03 & 0,0031 & 0,014 & $0,0-26$ & 0,0006 \\
\hline Pinus murrayana ....... & $0,99_{1}^{\prime}$ & 0,26 & $(1,2,2$ & 0,17 & 0,03 & 0,0045 & 0,030 & 0,030 & $0,001 \div$ \\
\hline Picea abies ........... & 0,81 & 0,27 & $(0,2 \cdot 2$ & $0, \geq 0$ & 0,07 & 0,0105 & 0,031 & 0,060 & 0,0009 \\
\hline Picea sitchensis ......... & 0,93 & 0,35 & $0,2 \cdot 2$ & 0,11 & 0,06 & 0,0425 & $0,022^{\prime}$ & 0,067 & 0,0011 \\
\hline Pseudotsuga mensiesii.... & $1,1,6$ & 0,50 & 0,18 & 0,18 & 0,07 & 0,0055 & 0,030 & 0,050 & 0,0009 \\
\hline Tisuga heterophylla ....... & $0,94^{\prime}$ & 0,31 & 0,20 & $0,1 \mathbf{1}^{\prime}$ & 0,07 & 0,0050 & 0,028 & $0,1^{\prime} t^{\prime}$ & 0,0011 \\
\hline \multicolumn{10}{|l|}{ Cones } \\
\hline Pinus sylvestris . ....... & 0,41 & 0,065 & 0,16 & 0,11 & $0,0 \cdot 2$ & 0,0050 & 0,040 & 0,016 & 0,0005 \\
\hline Pinus murrayana ....... & 0,28 & 0,025 & 0,11 & 0,05 & $0,0: 2$ & 0,0015 & 0,011 & 0,016 & $0,000^{\prime} t$ \\
\hline Pinus nigra laricio . . . . . . & 0,28 & 0,030 & 0,10 & 0,13 & $0,0.2$ & 0,0030 & 0,010 & 0,016 & $0,000^{\prime}$ \\
\hline
\end{tabular}

En ce qui concerne les autres éléments, on observe peu de différences pour le sodium (sauf pour Tsuga heterophylla qui a une teneur 2 fois plus élevée) et pour le fer (Tsuga heterophylla et Pseudotsuga menziesii ont cependant une teneur presque double). Pour le manganèse, il faut noter chez Abies grandis, Pseudotsuga menziesii et Picea sitchensis des valeurs environ ro fois plus élevées que chez les autres essences. Enfin pour le cuivre, il n'y a pratiquement pas de différence, toutes les espèces étant très pauvres en cet élément, sans doute à cause du substrat granitique carencé en cuivre.

\section{b) Variations des teneurs en éléments au cours de l'année.}

LuTZ et CHANDLER (I946) notent que les teneurs des feuilles en azote, phosphore et potassium diminuent tout au long de la période de croissance avec un maximum at printemps et un minimum en automne, alors qu'au contraire le taux de calcium augmente. Pour les litières, Owen (I954) sur Picea sitchensis et Aussenac, Bonneau, et LE TACON (1972) sur Pinus sylvestris, Picea abies et Abies grandis, constatent les mêmes faits, ces derniers auteurs précisant qu'ils sont particulièrement nets chez Pinus sylvestris.

Si nous examinons les variations des teneurs en éléments biogènes des aiguilles de la litière au cours des 4 trimestres (tab1. 3), nous arrivons aux mêmes conclusions 
TABLEAU 5

Apport au sol d'éléments minéraux (en $\mathrm{kg} / \mathrm{ha} / \mathrm{an}$ ) par l'intermédiaire des aiguilles

Mineral supply to the soil $(\mathrm{kg} / \mathrm{ha} / \mathrm{year})$ by needle litter fall

\begin{tabular}{|c|c|c|c|c|c|c|c|c|c|c|c|}
\hline Essence & Année & $\mathrm{N}$ & $\mathrm{Ca}$ & $\mathrm{K}$ & $\mathrm{Mg}$ & $\mathrm{P}$ & $M n$ & $\mathrm{Na}$ & $\mathrm{Fe}$ & $\mathrm{Cu}$ & Total \\
\hline \multirow{5}{*}{ Pinus sylvestris } & 1971 & 84,13 & 35,17 & $\mid 1,81$ & 5,54 & 5,92 & 2,60 & 1,65 & 1,69 & 0,1062 & 151,572 \\
\hline & 1972 & 69,10 & 30,08 & 12,53 & 5,28 & 6,27 & 2,15 & 1,18 & 1,32 & 0,066 & 128,576 \\
\hline & 1973 & 45,93 & 31,08 & $2 \cdot 2,2 \cdot 2$ & 5,30 & 4,19 & 2,19 & 1,35 & 1,65 & 0,060 & 113,970 \\
\hline & 197' & 80,41 & 35,92 & 16,47 & 6,80 & 5,66 & 2,02 & 2,28 & 1,85 & $0,06^{\prime} t$ & 151,474 \\
\hline & Moyenne & 69,97 & $3 \cdot 3,06$ & 16,51 & 5,73 & $.5, j 1$ & 2,21 & 1,69 & $1,6.3$ & $0,06.3$ & 136,398 \\
\hline \multirow{5}{*}{ Pinus nigra laricio } & 1971 & 14,28 & 19,69 & 7,79 & 3,86 & 3,01 & 0,70 & 1,01 & 1,12 & $0,0<9$ & 81,509 \\
\hline & 1972 & 11,16 & 20,50 & $10,0_{t}^{\prime}$ & 5,44 & 3,51 & 0,80 & $1, \dot{\prime}+1$ & 1,13 & 0,057 & $84,0.37$ \\
\hline & 1973 & $\geq 6,23$ & 18,73 & $10,5: 2$ & 4,97 & 2,07 & 0,77 & 1,07 & 1,21 & 0,051 & 65,621 \\
\hline & 1974 & $53,5 \div$ & 32,16 & 12,50 & 7,51 & $4,2 ' t$ & 0,87 & 2,32 & 1,61 & 0,060 & 114,790 \\
\hline & Moyenne & 41,30 & 22,77 & 10,21 & $5,4: 5$ & 3,21 & 0,79 & $1,4: 5$ & $1,2 \gamma$ & $0,0.54$ & 86,489 \\
\hline \multirow{5}{*}{ Pints murrayanas } & 1971 & 45,47 & 18,35 & 13,60 & 4,21 & 3,81 & 1,99 & 1,57 & 1,27 & 0,043 & 90,313 \\
\hline & 1972 & 53,66 & 26,16 & 13,53 & 4,97 & 4,80 & 2,48 & 1,76 & 1,53 & 0,062 & 108,952 \\
\hline & 1973 & 33,07 & 18,69 & 18,09 & 4,11 & $\because, 59$ & 1,99 & 1,19 & 1,68 & 0,056 & 81,766 \\
\hline & 1974 & $59, \geq 6$ & 30,52 & $15,8: 3$ & 5,59 & 5,15 & 3,18 & 2,43 & $2,2 \cdot 2$ & 0,079 & 121,259 \\
\hline & Ioyenne & 47,87 & 23,43 & 15,26 & 4,72 & $t, 09$ & $2,+1$ & 1,81 & 1,68 & 0,060 & $101,32: 3$ \\
\hline \multirow{5}{*}{ Picea abies } & 1971 & 65,90 & $\underline{2}^{\prime}, 6.3$ & 9,03 & 8,39 & 5,21 & 1,30 & 1,53 & 1,51 & 0,057 & 117,557 \\
\hline & 1972 & 112,76 & $4: 2,93$ & 26,71 & 19, is & 10,26 & $2,01_{1}^{\prime}$ & 像: & 2,50 & 0,145 & 221,25 \\
\hline & 1973 & $2 \cdot 2,210$ & 8,49 & $5, X^{\prime}+$ & 5,17 & 1,91 & 0,31 & $0,9: 3$ & $0,7\}$ & $0,03: 3$ & 45,623 \\
\hline & 197' & $\therefore 2,1$ & 17,30 & $11,0,5$ & 7,71 & $4,7 x$ & $(1,+i ;)$ & 1,91 & 1,36 & 0,053 & $96,19: 3$ \\
\hline & Moyenne & $6.3,32$ & $2: 3,31$ & 12,91 & $10,1^{9}$ & $-j$, il & $1,0 \tau$ & 2,20 & 1, , :3 & 0,072 & $120,1: 7$ \\
\hline
\end{tabular}




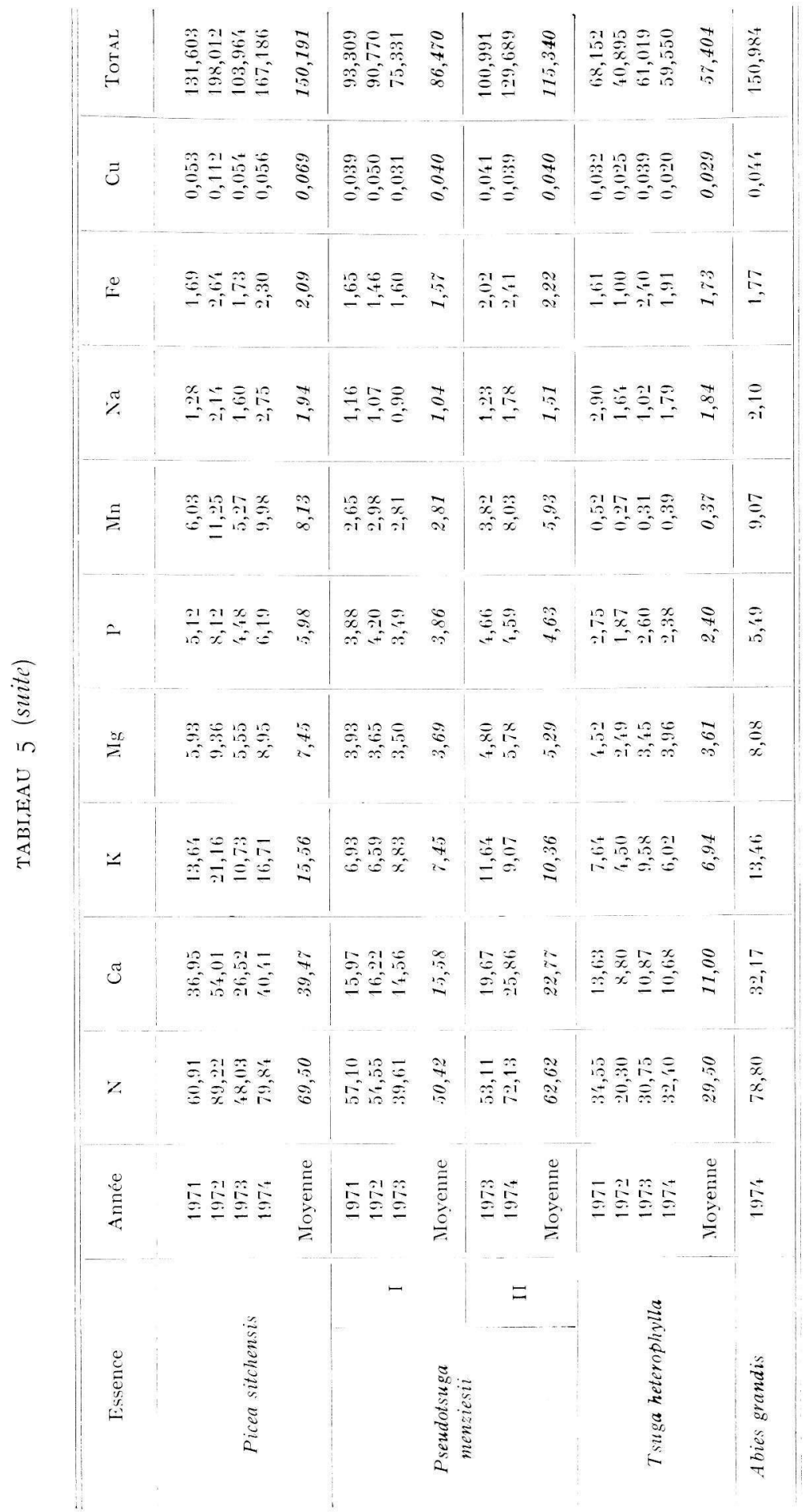


chez les Pins pour l'azote, le phosphore et le potassium, mais les résultats sont moins nets pour le calcium. Chez les autres espèces, certains éléments confirment cette observation, mais il est impossible de généraliser ; parfois même les résultats obtenus sont inverses, ainsi chez Tsuga heterophylla l'azote et le phosphore ont une teneur minimale au printemps alors que le calcium a son pourcentage minimal en automne.

Les variations des teneurs en magnésium sont difficiles à interpréter, celles-ci sont généralement plus faibles en automne.

Ėn ce qui concerne le fer, les pourcentages sont souvent élevés au printemps et faibles en automne; quant aux sodium et manganèse, ils ont généralement des valeurs fortes en automne ou en hiver; enfin pour le cuivre, les teneurs ne varient pas de façon nette et ordonnée au cours des 4 trimestres.

\section{III. - Apport d'éléments minéraux au sol par les litières}

Le retour annuel moyen d'éléments minéraux au sol par les aiguilles des différentes espèces (tab1. 5), calculé par trimestre, est très variable puisqu'il va de $57 \mathrm{~kg} /$ ha pour Tsuga heterophylla à plus de $\mathrm{I} 5 \mathrm{O} \mathrm{kg} / \mathrm{ha}$ pour Abies grandis et Picea sitchensis ${ }^{(1)}$.

En fonction de cet apport total d'éléments par les aiguilles, les espèces se classent dans l'ordre suivant :

\section{Abies grandis > Picea sitchensis > Pinus sylvestris > Picea abies > Pseudotsuga menziesii II > Pinus murrayana > Pinus nigra laricio > Pseudotsuga menziesii I > Tsuga heterophylla.}

Remarquons la différence entre les deux peuplements de Pseudotsuga menziesii, de même âge, mais d'origines différentes.

Ein I973, année de faible production de litière, le retour d'éléments au sol est aussi moins important chez toutes les espèces (une exception : Tsuga heterophylla où la chute de litière fut inférieure en I972).

Pour les brindilles et les cônes, les apports en éléments ont été calculés annuellement à partir des teneurs moyennes reportées dans le tableau 4 ; pour les divers, dominés par les débris d'aiguilles, nous avons utilisé pour les calculs par trimestre, les teneurs des aiguilles. Nous n'avons pas tenu compte de l'apport minime (tabl. 2) des brindilles d'Abies grandis et des cônes de Picea sitchensis et Tsuga heterophylla.

Les résultats obtenus concernant l'apport en éléments minéraux par la litière totale sont regroupés dans le tableau 6.

Cette fois les espèces se classent dans l'ordre suivant en fonction de l'apport total par la litière :

\section{Pinus sylvestris $>$ Abies grandis $>$ Picea sitchensis $>$ Picea abies $>$ Pinus murrayana $>$ Pseudotsuga menziesii II $>$ Pimus nigra laricio Pseudotsuga menziesii I > Tsuga heterophylla.}

L'influence des brindilles, cônes et divers est donc très nette, surtout pour Pinus sylvestris et Pinus murrayana. 


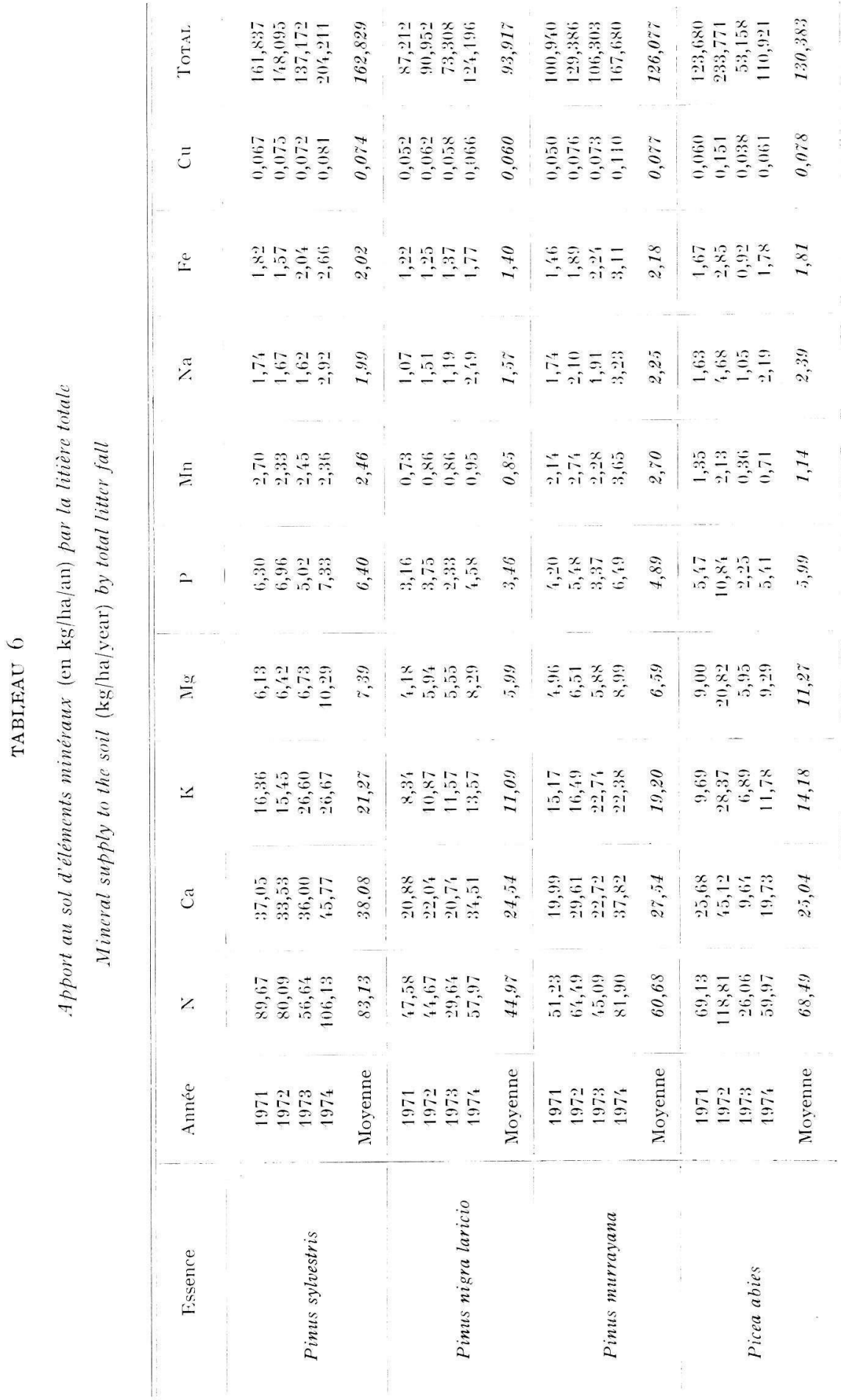




\begin{tabular}{|c|c|c|c|c|c|c|c|c|c|}
\hline$\stackrel{\vec{t}}{\stackrel{4}{0}}$ & 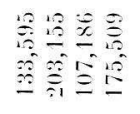 & $\begin{array}{l}\overrightarrow{0} \\
\infty \\
0 \\
0\end{array}$ & 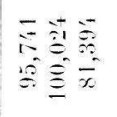 & $\begin{array}{l}0 \\
0 \\
0 \\
3 \\
3\end{array}$ & 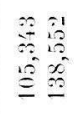 & $\begin{array}{l}\infty \\
\text { in } \\
\text { in } \\
-1\end{array}$ & 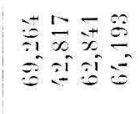 & 䒺 & 产 \\
\hline $\bar{U}$ & $\begin{array}{l}200 \\
=0=0\end{array}$ & E & $\begin{array}{l}\overline{0} \\
=0 \\
=0\end{array}$ & $\stackrel{8}{:}$ & 等 & $\underset{0}{0}$ & 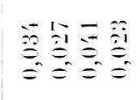 & $\stackrel{i}{\therefore}$ & $=$ \\
\hline : & 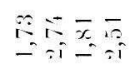 & $\stackrel{s}{i}$ & 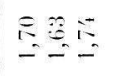 & $\underset{8}{8}$ & 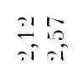 & $\therefore$ & 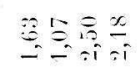 & $\hat{\alpha}$ & $\hat{x}$ \\
\hline$\tilde{z}$ & 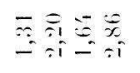 & $\stackrel{8}{\therefore i}$ & $\begin{array}{l}=x-5 \\
=0\end{array}$ & $\begin{array}{l}= \\
=\end{array}$ & $\stackrel{x}{x}$ & $\stackrel{B}{-1}$ & 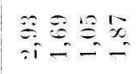 & $\stackrel{8}{-1}$ & $\stackrel{\bar{z}}{i i}$ \\
\hline$\Xi$ & $\begin{array}{l}=\bar{x} \\
=0 \\
=0 \\
=0\end{array}$ & 落 & 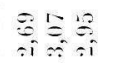 & $\begin{array}{l}8 \\
\therefore i\end{array}$ & 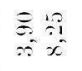 & 8 & 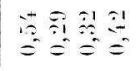 & $\stackrel{\vec{\alpha}}{i}$ & 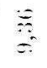 \\
\hline 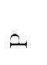 & 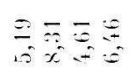 & $\frac{\pi}{6}$ & $\Leftrightarrow$ & 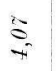 & 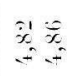 & $\neq$ & $\begin{array}{ccc}\bar{x} & 0 \\
-i & 0\end{array}$ & $\stackrel{9}{i}$ & 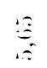 \\
\hline$\stackrel{x}{z}$ & $\begin{array}{l}13 \pi \\
=0 \\
=0\end{array}$ & $\therefore$ & $\triangleq \overline{1}$ & $\vec{a}$ & 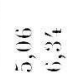 & $\therefore$ & $=$ & $\therefore$ & 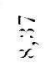 \\
\hline$\therefore$ & $\begin{array}{l}2: 3 \\
0-1=-15\end{array}$ & $\stackrel{2}{-2}$ & $\begin{array}{l}0:= \\
\therefore \therefore=0\end{array}$ & $\therefore$ & 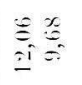 & $\begin{array}{l}2 \\
2 \\
2\end{array}$ & $\begin{array}{l}19=0.8 \\
\therefore=0 \\
\therefore=0\end{array}$ & 5 & $\bar{\vdots}$ \\
\hline$\tilde{s}$ & 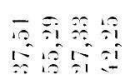 & $\stackrel{8}{8}$ & $\begin{array}{l}1 \\
\vdots 0 \\
0=0\end{array}$ & ?ֶ: & 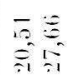 & 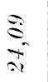 & $\begin{array}{l}x=0 \\
x=0 \\
0=0\end{array}$ & $\stackrel{P}{=}$ & 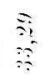 \\
\hline$\angle$ & 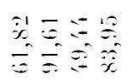 & $\sum$ & 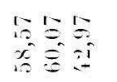 & $\grave{3}$ & 合帘 & $\begin{array}{l}7 \\
8\end{array}$ & 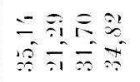 & E & $\stackrel{-1}{\approx}$ \\
\hline$\stackrel{g}{\Xi}$ & 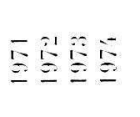 & 竎 & 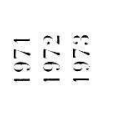 & 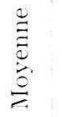 & $\stackrel{0}{0}$ & 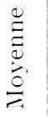 & 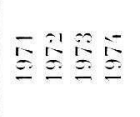 & 兰 & 窝 \\
\hline 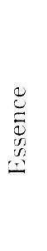 & 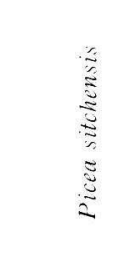 & & & 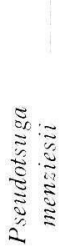 & $=$ & & 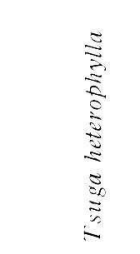 & & 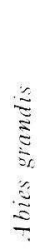 \\
\hline
\end{tabular}


Comparées aux données de la littérature (voir notamment RoDr et BAzILEVICH, I967) nos valeurs sont relativement élevées en raison de l'importance des chutes de litière. Eilles sont généralement plus fortes que celles de Viro (I955) pour Pinus sylvestris et Picea abies, de Mork (1942) et Bonnevie-Svendsen et GjEms (I957) pour les mêmes espèces, de Ovington et MadGwick (I959) pour Pinus sylvestris, de Nihlgånd (I972) pour Picea abies (sauf pour le manganèse et le fer), de Aussenac, Bonneau et Le Tacon (I972) pour Pimus sylvestris, Picea abies et Abies grandis (sauf pour le calcium), de TARrant, ISAaC et CHANdLER (I95I) pour Picea sitchensis et Pseudotsuga menziesii, de OWEN (I954) pour Picea sitchensis et de Heilman et Gesser. (I963) pour Pseudotsuga menziesii.

\section{CONCLUSION}

La quantité moyenne d'aiguilles retournant au sol annuellement est très variable d'une espèce à l'autre puisqu'elle va de $2,8 \mathrm{t}$ /ha pour Tsuga heterophylla à $6,8 \mathrm{t}$ pour Picea sitchensis; quant à la production de litière totale, elle est comprise entre 2,9 t/ha/an pour Tsuga heterophylla et 8,6 t pour Pinus murrayana.

Seul le peuplement de Tsuga heterophylla a une production moyenne de litière totale inférieure à celle de la Hêtraie étudiée dans cette même forêt de Villecartier (Gloaguex et ToufFeit, I974).

Une chute de litière saisonnière a pu être mise en évidence pour Pinus sylvestris, P. nigra laricio, P. murrayana, Abies grandis, Pseudotsuga menziesii et Tsuga heterophylla, par contre elle est très irrégulière chez Picea abies et $P$. sitchensis.

En ce qui concerne le retour d'éléments minéraux au sol par l'intermédiaire de la litière totale, il existe aussi de très grosses différences entre les espèces. Ainsi l'apport moyen minimal est, pour Tsuga heterophylla, de $30,7 \mathrm{~kg} / \mathrm{ha} /$ an d'azote, I I,4 $\mathrm{kg}$ de calcium, 7,2 $\mathrm{kg}$ de potassium, 3,8 kg de magnésium, 2,5 kg de phosphore, $0,4 \mathrm{~kg}$ de manganèse, $0,03 \mathrm{~kg}$ de cuivre; il est, pour un des peuplements de Pseudotsuga menziesii, de I, I kg de sodium et enfin pour Pinus nigralaricio de I,4 $\mathrm{kg}$ de fer. L, 'apport moyen maximal est de $83, \mathrm{I} \mathrm{kg} / \mathrm{ha} / \mathrm{an}$ d'azote, $2 \mathrm{I}, 3 \mathrm{~kg}$ de potassium et $6,4 \mathrm{~kg}$ de phosphore chez Pinus sylvestris, de 40,6 kg de calcium chez Picea sitchensis, de II,3 $\mathrm{kg}$ de magnésium et $2,4 \mathrm{~kg}$ de sodium chez Picea abies, de 9,4 kg de manganèse chez Abies grandis, de $2,4 \mathrm{~kg}$ de fer pour un des peuplements de Psendotsuga menziesii et enfin de $0,08 \mathrm{~kg}$ de cuivre pour Picea abies et Pinus murrayana.

La restitution totale d'éléments au sol varie de $59,8 \mathrm{~kg} / \mathrm{ha} / \mathrm{an}$ pour Tsuga heterophylla à $\mathrm{I} 62,8 \mathrm{~kg}$ pour Pinus sylvestris, elle est de $86 \mathrm{~kg}$ pour la Hêtraie (Gloaguex et ToufFe'T, I974).

Enfin, le retour d'éléments au sol par l'intermédiaire des aiguilles est compris entre $57,4 \mathrm{~kg} / \mathrm{ha} / \mathrm{an}$ pour Tsuga heterophylla et $\mathrm{I} 5 \mathrm{I}, \mathrm{O} \mathrm{kg}$ pour Abies grandis; il est de $70-74 \mathrm{~kg}$ par les feuilles de Hêtre. 


\section{SUMMARY}

\section{IITTER PRODUCTION AND ITS MINERAL SUPPLY TO THE SOIL IN SOME CONIFEROUS STANDS IN BRITTANY (FRANCE)}

Yearly production of litter and its mineral supply to the soil (nitrogen, calcium, potassium, magnesium, phosphorus, manganese, sodium, iron and copper) have been determined in artificial stands of 8 conifer species from 20 to 33 years old: Pinus sylvestris, Pinus nigra laricio, Pinus murrayana, Picea abies, Picea sitchensis, Pseudotsuga menziesii, Tsuga heterophylla and Abies grandis, during 4 years: 1971, 1972, 1973 and 1974.

Average yearly needle fall is very variable from one species to an other whereas it varies from $2.8 \mathrm{t} / \mathrm{ha}$ for Tsuga heterophylla to $6.8 \mathrm{t}$ for Picea sitchensis; total litter production ranges from 2.9 t/ha/year for Tsuga heterophylla to $8.6 \mathrm{t}$ for Pinus murrayana.

Only Tsuga heterophylla has an average total litter production inferior to the beech forest one (Gloaguen and Touffet, I974).

A seasonal litter fall can be observed for Pinus sylvestris, P. nigra lavicio, P. murrayana, Abies grandis, Pseudotsuga menziesii and Tsuga heterophylla; on the other hand it is very irregular for Picea abies and P. sitchensis.

The mineral return to the soil by the total litter is very different according to the species. So, the mean minimum supply is, for Tsuga heterophylla, $30.7 \mathrm{~kg} / \mathrm{ha} /$ year of nitrogen, II. $4 \mathrm{~kg}$ of calcium, $7.2 \mathrm{~kg}$ of potassium, $3.8 \mathrm{~kg}$ of magnesium, $2.5 \mathrm{~kg}$ of phosphorus, $0.4 \mathrm{~kg}$ of manganese, $0.03 \mathrm{~kg}$ of copper; it is, for one of the stands of Pseudotsuga menziesii, I. I kg of sodium and lastly, for Pinus nigra laricio, I.4 $\mathrm{kg}$ of iron. The mean maximum supply is $83 . \mathrm{I} \mathrm{kg} / \mathrm{ha} / \mathrm{year}$ of nitrogen, $2 \mathrm{I} .3 \mathrm{~kg}$ of potassium and $6.4 \mathrm{~kg}$ of phosphorus for Pinus sylvestris, $40.6 \mathrm{~kg}$ of calcium for Picea sitchensis, II.3 kg of magnesium and $2.4 \mathrm{~kg}$ of sodium for Picea abies, $9.4 \mathrm{~kg}$ of manganese for Abies grandis, $2.4 \mathrm{~kg}$ of iron for one of the stands of Psendotsuga menziesii and lastly $0.08 \mathrm{~kg}$ of copper for Picea abies and Pinus murrayana.

The total return of nutrients to the soil changes from $598 \mathrm{~kg} / \mathrm{ha} /$ year for Tsuga heterophy'la to $162.8 \mathrm{~kg}$ for Pinus sylvestris, it is $86 \mathrm{~kg}$ for the beech forest (GLOAGuen and TOUFFET, I974).

Lastly, the mineral supply to the soil by the needle litter varies from $57.4 \mathrm{~kg} / \mathrm{ha} / \mathrm{vear}$ for Tsuga heterophylla to I $5 \mathrm{I} . \mathrm{O} \mathrm{kg}$ for Abies grandis; it is $70-74 \mathrm{~kg}$ for the beech leaf litter fall.

\section{RÉFÉRENCES BIBLIOGRAPHIQUES}

Andicott F. T., Lyon J. L., I973. Physiological ecology of abscission. In shedding of plant parts by T. T. Kozlowsky, Academic Press, New York and London, 85-124.

Alvera B., i973. Estudios en bosques de coniferas del Pirineo Central. Serie A: Pinar con acebo de San Juan de la Peña. I. Producción de hojarasca. Pirineos, 109, I y-29.

Aussenac G., 1969. Production de litière dans divers peuplements forestiers de l'list de la lirance. Cicol. Plant., 4, 225-236.

Aussenac G., Bonneau M., Le Tacon F., 1972. Restitution des éléments minćraux au sol par l'intermédiaire de la litière et des précipitations dans quatre peuplements forestiers de l'lsst de la France. Qicol. Plant., $7, \mathrm{I}-2 \mathrm{I}$.

Bonnevie-Svenden C., Gjems O., 1957. Amount and chemical composition of the litter from Larch, Beech, Norway Spruce and Scots Pine stands and its effect on the soil. Medd. Norske Skogforsøkst., 48, II I-I 74 .

Bornebusch C. H., I937. Chute d'aiguilles naturelle d'Épicea (en danois). Forstl. Forsogsí. Manm., $14,173-176$.

Bray J. R., Gorham E., 1964. Litter production in forests of the world. Adv. Ficol. Research, 2, IOI-I57.

Carlisle A., Brown A. H. F., White E. J., ig66. Litter fall, leaf production and the effects of defoliation by Tortrix viridana in a sessile oak (Quercus petraea) woodland. J. Ecol., 54, 65-85.

Chander R. F., r944. Amount and mineral nutrient content of freshly fallen needle litter of some north-eastern conifers. Proc. Soil Sci. Soc. Amer., 8, 409-4II.

Cole D. W., Gessel S. P., Dice S. F., I967. Distribution and cycling of nitrogen, phosphorus, potassium and calcium in a second-growth Douglas-Fir ecosystem. In Primary productivity and mineral cycling in natural ecosystems, by H. E. Young, University of Maine Press, Orono, I97-232.

Delecour F., Weissen F., Nanson A., i967. Aspects techniques de l'échantillonnage des retombées annuelles et des horizons holorganiques des sols de forêts. Bull. Rech. agron. Gembloux, 2, 429-449. 
Duval L., I962. Dosage céruléomolybdique de l'acide phosphorique dans les sols, les végétaux et les engrais. Ann. agron., 13, 469-482.

DUVigneaud P., DenaeYer-DE Smet S., i97o. Biological cycling of minerals in temperate deciduous forests. In A nalysis of temperate forest ecosystems, by D. E. Reichle, Springer, Berlin Heidelberg New York, I99-225.

Esteoule J., Guvader J., Touffet J., ig7r. Les sols de la forêt de Villecartier. Bull. Ass. fr. Et. Sol, 2, 29-46.

Glonguen J. C., Touffet J., i974. Production de litière et apport au sol d'éléments minéraux dans une hêtraie atlantique. Ecol. Plant., 9, I I-28.

Gossuin A., I974. Production de litière dans une hêtraie et une pessière de Haute-Ardenne. Bull. Soc. roy. Bot. Belg., 10\%, 9-22.

Guittet J., I967. Composition et évolution de la litière de Pins silvestres en peuplements ouverts sur pelouse xérophile. Ecol. Plant., 2, 43-62.

Heilman P. E., Gessel S. P., I963. Nitrogen requirements and the biological cycling of nitrogen in Douglas-Fir stands in relationship to the effects of nitrogen fertilization. Plant and Soil, 18, 386-402.

LANier L., I968. Contribution à l'étude du rouge cryptogamique du Pin sylvestre dû au Lophoder mium pinastri (Schrad.) Chev. Étude phénologique sur la chute des aiguilles et sur la maturation des hystérothécies. Ann. Sci. forest., 25, 35-50.

Larivière G., Verdou J. P., I969. Contribution à l'étude du climat de la Bretagne. Monographie de la Météorologie nationale, Paris, $73,75 \mathrm{p}$.

Lemée G., Bichaut N., ig7i. Recherches sur les écosystèmes des réserves biologiques de la forêt de Fontainebleau. I. Production de litière et apport au sol d'éléments minéraux majeurs. GEcol. Plant., 6, I33-149.

Lossaint P., Rapp M., I97I. Répartition de la matière organique, productivité et cycles des éléments minéraux dans des écosystèmes de climat méditerranéen. Productivité des écosystèmes forestiers, Actes Coll. Bruxelles, I969, Unesco Paris, 597-617.

Lutz H. J., Chandler R. F., i946. Forest soils. John Wiley, New York, I vol, 5I4 p

Miller R. B., I968. Flows and cycles of macro- and micro-elements in a forest soil and its environment. Trans. 9 th int. Congr. Soil Sci., 4, 323-331.

Mork E., 1942. Über den Streufall in unseren Wäldern (en norvégien). Medd. Norske Skogforsoksv., 29, $297-365$.

Nihlgåd B., r972. Plant biomass, primary production and distribution of chemical elements in a beech and a planted spruce forest in South Sweden. Oikos, 23, 69-8I.

Ovington J. D., I959. The circulation of minerals in plantations of Pinus sylvestris L. Ann. Bot., 23, 229-239

Ovington J D., i962. Quantitative ecology and the woodland ecosystem concept. Adr. Ecol. Research, 1, $103-192$.

Ovington J. D., Madgwick H. A. I., I959. Distribution of organic matter and plant nutrients in a plantation of Scots Pine. Forest. Sci., 5, 344-355.

OWEN T. H., I954. Observations on the monthly litter-fall and nutrient content of Sitka Spruce litter. Forestry, 2\%, 7-15.

RApp M., I967. Production de litière et apport au sol d'éléments minéraux et d'azote dans un bois de Pins d'Alep (Pinus halepensis Mill.). OEcol. Plant., 2, 325-338.

RAPP M., I969. Production de litière et apport au sol d'éléments minéraux dans deux écosvstèmes méditerranéens : la forêt de Quercus ilex L. et la garrigue de Quercus coccifera L. (Ecol. Plant., 4, 377-4 IO.

Rodin L. E., Bazilevich N. I., I967. Production and mineral cycling in terrestrial vegetation. Oliver and Boyd, Edinburgh and London, I vol., $288 \mathrm{p}$.

Snedecor G. W., Cochran W. G., I97I. Méthodes statistiques. Association de coordination technique agricole, Paris, I vol., 649 p.

Tarrant R. F., Isaac L. A., Chandler R. F., i95i. Observations on litter fall and foliage nutrient content of some Pacific Northwest tree species. J. For., 49, 9I4-9I5.

Touffet J., 1964. Aperçu bryologique sur la forêt de Villecartier (Ille-et-Vilaine) : Les Sphaignes. Bull. Soc. sci. Bretagne, 39, 69-75.

Touffet J,, I97I. Contribution à l'étude de la bryoflore de la forêt de Villecartier (Ille-et-Vilaine) Botanica Rhedonica, sér. A, 11, 189-222.

Toutain F., Duchaufour P., i97o. Étude comparée des bilans biologiques de certains sols de hêtraie. Ann. Sci. forest., 27, 39-61.

Tsutsumi T., i97r. Accumulation and circulation of nutrient elements in forest ecosystems. Productivity of forest ecosystems. Proc. Brussels Symp., I969, Unesco Paris, 543-552.

Ulrich B., MAYER R., r973. Systemanalyse des Bioelement-Haushalts von Wald-Ökosystemen. In Ökosystemforschung, von H. ElLenberG, Springer, Berlin Heidelberg New York, I65-I74

Viro P. J., I955. Investigations on forest litter. Commun. Inst. for. Finl., 45, I-65.

Will G. M., I968. The uptake, cycling and removal of mineral nutrients by crops of Pimus radiata. Proc. N. Z. Ecol. Soc., 15, 20-24. 